\title{
Ductal carcinoma in situ of the breast: correlation between histopathological features and age of patients
}

Amanda Arantes Perez, Débora Balabram, Marcio de Almeida Salles and Helenice Gobbi*

\begin{abstract}
Background: The histopathological subtype, nuclear grade and presence or absence of comedonecrosis are established as critical elements in the reporting of ductal carcinoma in situ (DCIS) of the breast. The aims of this study were to determine the frequencies of morphological subtypes of DCIS, nuclear grade and comedonecrosis; to compare the age of patients with the histopathological characteristics of DCIS, and to assess the agreement of grade between in situ and invasive components in DCIS cases that were associated with invasive carcinoma.
\end{abstract}

Methods: We evaluated a series of 403 cases of DCIS, pure or associated with invasive mammary carcinoma, consecutively identified from the histopathology files of the Breast Pathology Laboratory, Federal University of Minas Gerais, Brazil, from 2003 to 2008.

Results: DCIS displayed a single growth pattern in most cases (55.1\%) and the solid subtype was the most common morphology (42.2\% of the total). High-grade DCIS was identified in 293/403 cases (72.7\%) and comedonecrosis was present in 222/403 cases (55\%). Among DCIS with a single architectural pattern, high grade was more common in the solid subtype $(151 / 168$ cases, $89.9 \% ; p<0.001)$. Only $32 \%$ of tumours with a cribriform pattern had high nuclear grade. Comedonecrosis was more common in the solid morphology than in the cribriform, papillary and micropapillary subtypes $(p<0.001)$. Patients with high-grade DCIS were younger in relation to patients with low-grade DCIS $(p=0.027)$ and patients with tumours with comedonecrosis were also younger in comparison to patients with tumours without comedonecrosis $(p=0.003)$. Fair agreement was observed between in situ and invasive components with regard to grade (weighted kappa $=0.23$ ).

Conclusions: The high nuclear grade and the presence of comedonecrosis were identified more frequently in younger patients and more often correlated with the solid pattern of DCIS.

Virtual Slides: The virtual slide(s) for this article can be found here: http://www.diagnosticpathology.diagnomx.eu/vs/ 13000_2014_227

Keywords: Ductal carcinoma in situ, Histological pattern, Morphology, Breast cancer

\section{Background}

Ductal carcinoma in situ of the breast (DCIS) is defined as a neoplastic proliferation of epithelial cells confined to the mammary ductal-lobular system and characterized by subtle to marked cytological atypia and an inherent but not necessarily obligate tendency for progression to invasive breast cancer [1]. DCIS constitutes $20 \%$ of all newly diagnosed breast cancer cases and $30 \%$ to $40 \%$ of

\footnotetext{
* Correspondence: hgobbi@medicina.ufmg.br

Breast Pathology Laboratory, School of Medicine, Federal University of Minas Gerais, Av. Prof. Alfredo Balena, 190, Belo Horizonte, Minas Gerais, Brasil
}

the breast cancer cases diagnosed mammographically in the United States of America [2,3].

Currently, it is accepted that DCIS is not one entity but rather a heterogeneous group of lesions with different clinical, radiological, morphological and genetic features $[4,5]$.

Genetic and molecular studies have been published aiming to establish prognostic and predictive factors and new targeted therapies to reduce the risk of recurrence and progression to invasive carcinoma in patients with DCIS [2,6-12]. These advances may become an important part 
of evaluating DCIS, but at this time, size, grade, comedonecrosis and margin status are established as critical elements in the reporting of DCIS $[1,13]$.

The purpose of this study was to determine the frequencies of DCIS subtypes, nuclear grade and comedonecrosis of a series of cases of DCIS of the breast; to compare the age of patients with the histopathological characteristics of DCIS, and to assess the agreement of grade between in situ and invasive components in DCIS cases that were associated with invasive carcinoma.

\section{Methods}

Four hundred and three cases of DCIS, pure or associated with invasive carcinoma, were consecutively identified from the histopathology files of the Breast Pathology Laboratory, School of Medicine, Federal University of Minas Gerais, Brazil, from 2003 to 2008. Histopathological sections from all cases were reviewed by two authors (HG and AAP) and data considered in the analysis was that obtained in the current slide review.

The criteria defined by World Health Organization (2012) were used for histopathological diagnosis, classification and grading of DCIS and invasive carcinoma [1]. The nuclear grade of DCIS was classified as low, intermediate or high on the basis of nuclear size, pleomorphism, chromatin pattern, presence of nucleoli and mitotic activity. DCIS with low nuclear grade consists of a monotonous population of cells with slightly enlarged nuclei, smooth nuclear membranes, finely dispersed chromatin, inconspicuous nucleoli and rare mitoses. The cells of low-grade DCIS are typically polarized with the longest axis of the cell oriented perpendicularly to the basement membrane, and radially distributed around neoformed extracellular lumina. DCIS of intermediate nuclear grade consists of a population of cells with intermediate-size nuclei with coarser chromatin and evident but still inconspicuous nucleoli. Mitotic activity may be encountered, but it is rarely high. The neoplastic cells may be polarized. DCIS of high nuclear grade consists of cells with large ( $>2.5$ times the size of a red blood cell) and pleomorphic nuclei that show vesicular and coarse chromatin, with prominent and often multiple and irregular nucleoli. The neoplastic epithelial cells usually lack polarity, and mitotic activity is easily detected $[1,14]$. The highest nuclear grade was used for the purpose of statistical analysis. The nuclear grade of DCIS was not evaluated in eleven cases $(2.7 \%)$ because the slides were not available for review. The grade of invasive carcinoma was classified as low, intermediate or high based on an assessment of tubule/gland formation, nuclear pleomorphism and mitotic count [1]. Tubular formation was assessed over the whole tumour; nuclear pleomorphism was evaluated in the area showing the highest degree of pleomorphism, and mitotic counting was performed in the area exhibiting most proliferation [1].

The architectural patterns of DCIS included solid, cribriform, micropapillary, papillary and apocrine [1]. DCIS was divided into single (when $>90 \%$ of the in situ tumor displayed one architectural growth pattern) and mixed (when the dominant pattern constituted $<90 \%$ of the in situ carcinoma) [15].

The term comedonecrosis, which is poorly defined in the literature, was applied when significant necrosis, creating an appearance similar to comedos, seen in cutaneous acne, was noted in ducts involved by DCIS $[15,16]$.

We measured the largest focus of all pure DCIS cases. Because not all slides from each case were available to be reviewed, we did not analyze margin status and size of DCIS in cases associated with invasive carcinoma.

Pearson's asymptotic test was used to compare proportions. The $t$ test was used to compare age means among the groups and the Bonferroni test was used to multiple comparisons between them. The weighted kappa test was used to assess the concordance between nuclear grade of DCIS and histological grade of invasive carcinoma. Kappa values in the range 0.21 to 0.40 demonstrated fair agreement [17]. A $p$ value $<0.05$ was considered statistically significant. This study was approved by the Research Ethics Committee of the Federal University of Minas Gerais (protocol ETIC 655/08).

\section{Results}

Pure DCIS was detected in $110 / 403$ cases $(27.3 \%$ of the total) and $293 / 403$ cases $(72.7 \%$ of the total) were DCIS associated with invasive carcinoma.

The mean age of patients at diagnosis was 54.1 years (standard deviation \pm 13.1 ). There was no significant difference in age between pure DCIS and DCIS associated with invasive carcinoma $(\mathrm{p}=0.814)$.

The mean size of pure DCIS was $15.2 \mathrm{~mm}$ (range: 2 to $100 \mathrm{~mm}$; standard deviation \pm 15.8 ). There was no significant difference between the size of pure DCIS and age $(p=0.938)$, neither between the size of pure DCIS and presence of comedonecrosis $(\mathrm{p}=0.732)$. We did not find a significant difference in size between single growth pattern and mixed growth pattern $(\mathrm{p}=0.213)$, and also between the size and the nuclear grade $(\mathrm{p}=$ 0.175 ). There was no significant difference between the size and the subtype of DCIS $(p=0.137)$. However, we identified a tendency of the micropapillary subtype to be larger than the other subtypes $(\mathrm{p}=0.06)$.

The frequencies of DCIS subtypes are shown in Table 1. Of the 403 cases of DCIS (pure and associated with invasive carcinoma), 222 cases (55.1\%) displayed a single growth pattern and 181 cases (44.9\%) showed a mixed growth pattern. Among architectural patterns, the solid subtype was the most common (170/403 cases; $42.2 \%)$. The 
Table 1 Frequencies of ductal carcinoma in situ subtypes

\begin{tabular}{lcc}
\hline Architectural pattern & $\mathbf{N}$ & $\%$ \\
\hline Solid* & 170 & 42.2 \\
Cribriform* $^{*}$ & 27 & 6.7 \\
Papillary* $^{*}$ & 12 & 3.0 \\
Micropapillary* & 13 & 3.2 \\
Apocrine* & 00 & 00 \\
Mixed & 181 & 44.9 \\
Total & 403 & 100 \\
\hline
\end{tabular}

*Single growth pattern.

pure micropapillary subtype was found in 13/403 cases (3.2\%). The apocrine morphology was not identified among DCIS with a single architectural pattern. There was no significant difference in single or mixed growth pattern between pure DCIS and DCIS associated with invasive carcinoma ( $\mathrm{p}=0.088)$.

The nuclear grade of DCIS was evaluated in 392/403 cases (97.3\%; Fig. 1A-C). High-grade DCIS was identified in 293/403 cases (72.7\%; Figure 1C). There was no significant difference in nuclear grade between pure DCIS and DCIS associated with invasive carcinoma $(p=0.142)$. The frequencies of different nuclear grades of DCIS and the relationship between nuclear grade and different single architectural patterns are respectively shown in Tables 2
Table 2 Frequencies of nuclear grade of ductal carcinoma in situ

\begin{tabular}{lcc}
\hline Nuclear grade & N & $\%$ \\
\hline Low grade & 38 & 9.4 \\
Intermediate grade & 61 & 15.2 \\
High grade & 293 & 72.7 \\
Missing & 11 & 2.7 \\
Total & 403 & 100 \\
\hline
\end{tabular}

and 3. Among DCIS with a single architectural pattern, high grade was more common in the solid subtype (151/ 168 cases, $89.9 \% ; \mathrm{p}<0.001)$. Only $32 \%$ of tumors with a cribriform pattern had high nuclear grade.

Comedonecrosis was present in 222/403 cases (55\%; Figure 1D). The frequencies of comedonecrosis in different architectural patterns are shown in Table 4. Among DCIS cases with a single growth pattern, comedonecrosis was more often in cases with a solid morphology $(\mathrm{p}<0.001)$. The cribriform, papillary and micropapillary subtypes more frequently did not present comedonecrosis $(\mathrm{p}<0.001)$.

Patients with high-grade DCIS were younger (mean age $=53$ years $)$ in relation to patients with low-grade DCIS (mean age $=58.4$ years; $\mathrm{p}=0.027$ ). Patients with tumours with comedonecrosis were also younger in

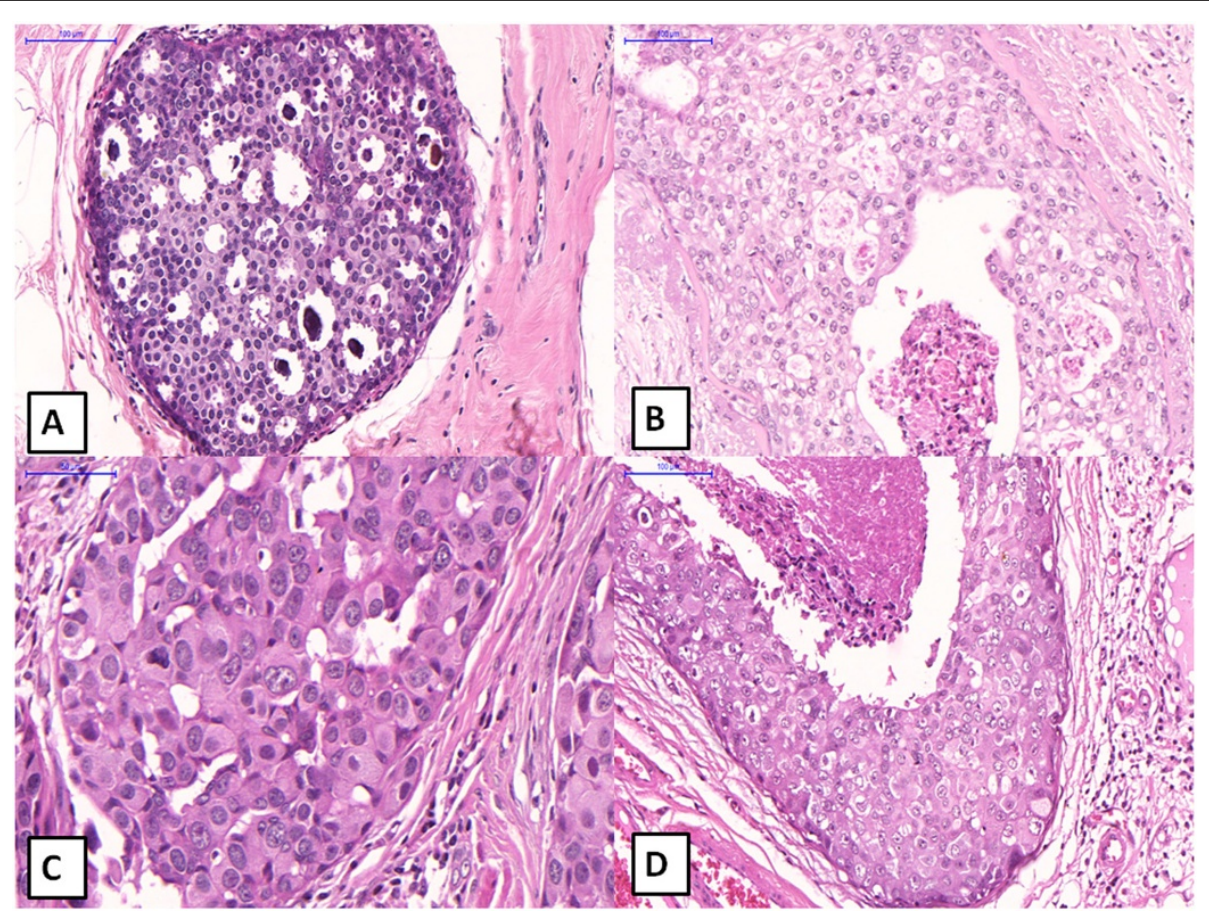

Figure 1 Ductal carcinoma in situ of the breast: different nuclear grades and comedonecrosis. A - Low grade ductal carcinoma in situ, cribriform type, showing uniform cells with mild atypia. Hematoxylin and eosin, x200. B - Intermediate grade ductal carcinoma in situ, showing cells with mild a moderate atypia and focal necrosis. Hematoxylin and eosin, x200. C - High grade ductal carcinoma in situ, solid type, showing severe atypical cells, without necrosis. Hematoxylin and eosin, x400. D - High grade ductal carcinoma in situ, solid type with extensive comedonecrosis. Hematoxylin and eosin, $x 200$. 
Table 3 Nuclear grade and architectural patterns of ductal carcinoma in situ

\begin{tabular}{llllll}
\hline Nuclear grade & Solid N (\%) & Cribriform N (\%) & $\begin{array}{l}\text { Architectural } \\
\text { Papillary N (\%) }\end{array}$ & $\begin{array}{l}\text { Pattern* } \\
\text { Micropapillary N (\%) }\end{array}$ & Total \\
\hline Low & $08(4.8)$ & $08(32.0)$ & $02(16.7)$ & $02(16.7)$ & $20(9.2)$ \\
Intermediate & $09(5.3)$ & $09(36.0)$ & $01(8.3)$ & $01(8.3)$ & $20(9.2)$ \\
High & $151(89.9)$ & $08(32.0)$ & $09(75.0)$ & $09(75.0)$ & $177(81.6)$ \\
Total & $168(100)$ & $25(100)$ & $12(100)$ & $12(100)$ & $217(100)$ \\
Missing & 02 & 02 & 00 & 01 & $p<0.001$ \\
\hline
\end{tabular}

*Single growth pattern; $p=$ significance level.

comparison to patients with tumours without comedonecrosis $(\mathrm{p}=0.003)$.

Invasive carcinoma of no special type was the most common (262/293 cases; $88.7 \%)$, followed by the tubular carcinoma (12/293 cases; $4.1 \%)$, mucinous carcinoma (07/293 cases; $2.4 \%)$, invasive lobular carcinoma (04/293 cases; $1.4 \%$ ), invasive papillary and micropapillary carcinomas $(03 / 293$ cases each one; $1 \%)$, secretory and cribriform carcinomas (02/293 cases each one; 0.7\%).

Fair agreement was observed between in situ and invasive components with regard to grade (weighted kappa $=0.23$ ).

\section{Discussion}

In the present study we evaluated morphological features of a series of DCIS pure (110 cases, $27.3 \%$ of the total) or associated with an invasive component (293 cases, $72.7 \%$ of the total) correlating them with the age of patients. Clinical and histological characteristics that may predict the risk of recurrence in women with DCIS include the age, tumour grade, presence of comedonecrosis, architectural subtype, tumour size, and width of resection margin $[18,19]$. In our series we evaluated nuclear grade, tumour size, comedonecrosis and age that are well established critical elements in the reporting of DCIS. Although recent genetic, molecular and immunohistochemical studies have contributed to advances in the understanding of the pathogenesis of DCIS, age and histopathological features remain the most established predictors of behavior in DCIS [1,8,20-26].

In our series, the majority of the cases (293 cases, $72.7 \%$ of the total) were high-grade DCIS; $15.2 \%$ of cases had an intermediate grade and 9.4\% were low-grade CDIS. The classification of DCIS has been based on the architectural growth pattern. Cytonuclear differentiation of tumour cells is more important than the architectural growth pattern and various novel classifications of DCIS have been proposed. Almost all modern classifications separate DCIS into three categories (high, intermediate, and low grade), but differ in the choice of features that are used for categorization [27]. Studies have reported nuclear grade to be the most significant predictor of recurrence on both univariate and multivariate analysis $[28,29]$. Therefore, cytonuclear grade has been used as the basic method for the assessment of intrinsic biological aggressiveness. Scripcaru and colleagues recognized, respectively, high-grade DCIS and intermediate nuclear grade in $45 \%$ and $41 \%$ of their cases [15]. These differences in the frequencies of grade are possibly attributed to the variation in the classification criteria, to the differences between the populations or to the detection method of the tumours (clinical or mammographically detected tumours).

We also correlated the nuclear grade of DCIS with the histological grade of invasive carcinoma to assess the agreement between in situ and invasive components in DCIS cases that were associated with invasive carcinoma. The fair agreement between in situ and invasive components with regard to grade (weighted kappa = 0.23 ) observed in our study is probably related to the various categories (grades 1, 2 or 3 ) included in the statistical analysis.

We showed that comedonecrosis was significantly more often in solid morphology than in cribriform, papillary and micropapillary subtype $(\mathrm{p}<0.001)$. Scripcaru et al. also identified a statistically significant difference between the presence of comedonecrosis in micropapillary and solid subtypes versus the cribriform morphology [15].

Table 4 Comedonecrosis and architectural patterns of ductal carcinoma in situ

\begin{tabular}{llllll}
\hline & & Architectural & & Pattern* & \\
Comedonecrosis & Solid N (\%) & Cribriform N (\%) & Papillary N (\%) & Micropapillary N (\%) & Total \\
\hline Absent & $36(21.2)$ & $21(77.8)$ & $09(75.0)$ & $08(61.5)$ & $74(33.3)$ \\
Present & $134(78.8)$ & $06(22.2)$ & $03(25.0)$ & $05(38.5)$ & $148(66.7)$ \\
Total & $170(100)$ & $27(100)$ & $12(100)$ & $13(100)$ & $222(100) p<0.001$ \\
\hline
\end{tabular}

*Single growth pattern; $\mathrm{p}=$ significance level. 
Comedonecrosis may be seen in association with any architectural pattern. The term "comedo DCIS" is widely used in historical series but does not confer either a specific grade or architecture to the lesion and there is no consensus in the literature regarding the amount of central necrosis required, so reproducibility as a category of DCIS is questionable [4]. Although rates of ipsilateral breast tumour recurrence are generally higher for tumours with a component of comedonecrosis than for those without, irrespective of adjuvant therapies, the presence of necrosis might be a weaker predictor of ipsilateral breast tumor recurrence than cellular architecture and nuclear grade [30].

The architectural patterns of DCIS include solid, cribriform, micropapillary, papillary and unusual variants (apocrine, signet ring, neuroendocrine, spindled, squamous or clear cells) [1]. Some combinations of nuclear grade and architectural pattern tend to be more frequent than others but any combination can occur [7]. The current World Health Organization classification does not recognize the low-grade (monomorphic) variant of DCIS with an exclusive flat type (clinging) pattern and did not recommend this terminology [1].

Our results showed that high grade was more common in the solid subtype $(89.9 \% ; \mathrm{p}<0.001)$ and only $32 \%$ of tumors with a cribriform pattern had high nuclear grade. Solid DCIS is characterized by a proliferation of neoplastic cells that fill, expand, and distort a duct. Nuclear grades can range from high to low in solid DCIS, although pure solid DCIS with low nuclear grade is fairly rare. Low-grade DCIS with solid architecture sometimes raises the differential diagnosis of lobular carcinoma in situ [1,31-33]. Cribriform DCIS is characterized by well-defined lumens lined by neoplastic cells. Such an orderly arrangement is rare in highgrade DCIS [7].

DCIS most frequently (62\%) shows a mixture of architectures, which is seen almost twice as often as the second most commonly seen pattern (solid, 31.9\%) [4]. In our series, $55.1 \%$ of cases displayed a single growth pattern and $44.9 \%$ showed a mixed growth pattern. Among architectural patterns, the solid subtype was more common (42.2\%). Similar to our results, Scripcaru and colleagues also described that $58 \%$ of cases of DCIS displayed a single growth pattern and $42 \%$ showed a mixed growth pattern [15].

Silverstein's group showed age to be an independent prognostic factor for local recurrence, which led to incorporation of age into the Van Nuys Prognostic Index by the division of patients into three groups ( $\geq 61$ years, $40-60$ years and $\leq 39$ years) [34]. Goldstein et al. examined pathologic features of DCIS in three different age groups of patients to identify differences that might explain why young patient age at the time of diagnosis is a risk for recurrence. They found that younger patients more frequently had higher nuclear grade DCIS, central necrosis, smaller initial biopsy maximum dimensions and close or positive margins [35].

In our series, patients with high grade DCIS were younger in relation to patients with low grade DCIS $(p=0.027)$ and patients with tumours with comedonecrosis were also younger in comparison to patients with tumours without comedonecrosis $(p=0.003)$. This group of younger patients may have an increased risk of local recurrence when treated with breast-conserving therapy due to a greater proportion of high nuclear grade DCIS and presence of comedonecrosis. In the present study, the type of treatment (radical or breast-conserving therapy) and the local recurrence were not evaluated. Maybe the association of high nuclear grade DCIS, comedonecrosis and younger patients can guide the clinicians to perform more aggressive surgical treatments, as these factors are associated with local recurrence.

\section{Conclusions}

The high nuclear grade and the presence of comedonecrosis were identified more frequently in DCIS of younger patients and more often correlated with the solid pattern DCIS. As clinical and histopathological features remain the most established predicts of behavior in DCIS, the choice of surgical treatment should be guide by them in addition to the size of DCIS and margin status.

\section{Consent}

Written informed consent was obtained from the patients for the publication of this report and any accompanying images.

\section{Abbreviation}

DCIS: Ductal carcinoma in situ.

\section{Competing interests}

The authors declare that they have no competing interests.

\begin{abstract}
Authors' contributions
AAP conceived the study, participated in the review of the original slides, and drafted the manuscript. MAS participated in the design of the study and helped drafting the manuscript. $D B$ performed the statistical analysis and helped drafting the manuscript. HG participated in the design and coordination of the study, reviewed the original slides, and helped drafting the manuscript. All authors have read and approved the final manuscript.
\end{abstract}

\section{Acknowledgments}

This work was supported in part by grants from Fundação de Amparo a Pesquisa de Minas Gerais (FAPEMIG), and Conselho Nacional de Desenvolvimento Científico e Tecnológico (CNPq).

Received: 24 August 2014 Accepted: 20 November 2014

Published online: 03 December 2014 


\section{References}

1. Lakhani SR, Ellis IO, Schnitt SJ, Tan PH, van de Vijver MJ, World Health Organization, International Agency for Research on Cancer: WHO Classification of Tumours of the Breast. 4th edition. IARC: Lyon; 2012.

2. Siziopikou KP: Ductal carcinoma in situ of the breast: current concepts and future directions. Arch Pathol Lab Med 2013, 137:462-466.

3. Bleicher RJ: Ductal carcinoma in situ. Surg Clin North Am 2013, 93:393-410.

4. Pinder SE: Ductal carcinoma in situ (DCIS): pathological features, differential diagnosis, prognostic factors and specimen evaluation. Mod Pathol 2010, 23:S8-S13.

5. Burstein HJ, Polyak K, Wong JS, Lester SC, Kaelin CM: Ductal carcinoma in situ of the breast. N Engl J Med 2004, 350:1430-1441.

6. Bane A: Ductal carcinoma in situ: what the pathologist needs to know and why. Int J Breast Cancer 2013, 2013:914053.

7. Ross DS, Wen YH, Brogi E: Ductal carcinoma in situ: morphology-based knowledge and molecular advances. Adv Anat Pathol 2013, 20:205-216.

8. Perez AA, Rocha RM, Balabram D, Souza AS, Gobbi H: Immunohistochemical profile of high-grade ductal carcinoma in situ of the breast. Clinics 2013, 68:674-678.

9. Nofech-Mozes S, Spayne J, Rakovitch E, Hanna W: Prognostic and predictive molecular markers in DCIS: a review. Adv Anat Pathol 2005, 12:256-264

10. Kerlikowske K, Molinaro AM, Gauthier ML, Berman HK, Waldman F, Bennington J, Sanchez H, Jimenez C, Stewart K, Chew K, Ljung BM, TIsty TD: Biomarker expression and risk of subsequent tumors after initial ductal carcinoma in situ diagnosis. J Natl Cancer Inst 2010, 102:627-637.

11. Lari SA, Kuerer HM: Biological Markers in DCIS and Risk of Breast Recurrence: A Systematic Review. J Cancer 2011, 2:232-261.

12. Rakovitch E, Nofech-Mozes S, Hanna W, Narod S, Thiruchelvam D, Saskin R, Spayne J, Taylor C, Paszat L: HER2/neu and Ki-67 expression predict non-invasive recurrence following breast-conserving therapy for ductal carcinoma in situ. Br J Cancer 2012, 106:1160-1165.

13. Lester SC, Bose S, Chen YY, Connolly JL, de Baca ME, Fitzgibbons PL, Hayes DF, Kleer C, O'Malley FP, Page DL, Smith BL, Tan LK, Weaver DL, Winer E: Protocol for the examination of specimens from patients with ductal carcinoma in situ of the breast. Arch Pathol Lab Med 2009, 133:15-25.

14. Pinder SE, Ellis IO: The diagnosis and management of pre-invasive breast disease: ductal carcinoma in situ (DCIS) and atypical ductal hyperplasia (ADH)-current definitions and classification. Breast Cancer Res 2003, 5:254-257.

15. Scripcaru G, Zardawi IM: Mammary ductal carcinoma in situ: a fresh look at architectural patterns. Int I Surg Oncol 2012, 2012:979521.

16. Jaffer S, Bleiweiss IJ: Histologic classification of ductal carcinoma in situ. Microsc Res Tech 2002, 59:92-101.

17. Landis JR, Koch GG: The measurement of observer agreement for categorical data. Biometrics 1977, 33:159-174.

18. Provenzano E, Hopper JL, Giles GG, Marr G, Venter DJ, Armes JE: Histological markers that predict clinical recurrence in ductal carcinoma in situ of the breast: an Australian population-based study. Pathology 2004, 36:221-229.

19. Ringberg $A$, Idvall I, Fernö $M$, Anderson $H$, Anagnostaki L, Boiesen $P$, Bondenson L, Holm E, Johanson S, Lindholm K, Ljungberg O, Ostberg G: Ipsilateral local recurrence in relation to therapy and morphological characteristics in patients with ductal carcinoma in situ of the breast. Eur J Surg Oncol 2000, 26:444-451.

20. Clark SE, Warwick J, Carpenter R, Bowen RL, Duffy SW, Jones JL: Molecular subtyping of DCIS: heterogeneity of breast cancer reflected in pre-invasive disease. Br J Cancer 2011, 104:120-127.

21. Gomes DS, Porto SS, Rocha RM, Gobbi H: Usefulness and limitations of E-cadherin and $\beta$-catenin in the classification of breast carcinomas in situ with mixed pattern. Diagn Pathol 2013, 8:114.

22. Bryan BB, Schnitt SJ, Collins LC: Ductal carcinoma in situ with basal-like phenotype: a possible precursor to invasive basal-like breast cancer. Mod Pathol 2006, 19:617-621.

23. Dabbs DJ, Chivukula M, Carter G, Bhargava R: Basal phenotype of ductal carcinoma in situ: recognition and immunohistologic profile. Mod Pathol 2006, 19:1506-1511.

24. Livasy CA, Perou CM, Karaca G, Cowan DW, Maia D, Jackson S, Tse CK Nyante S, Millikan RC: Identification of a basal-like subtype of breast ductal carcinoma in situ. Hum Pathol 2007, 38:197-204.
25. Paredes J, Lopes N, Milanezi F, Schmitt FC: P-cadherin and cytokeratin 5: useful adjunct markers to distinguish basal-like ductal carcinomas in situ. Virchows Arch 2007, 450:73-80

26. Tamimi RM, Baer HJ, Marotti J, Galan M, Galaburda L, Fu Y, Deitz AC, Connolly JL, Schnitt SJ, Colditz GA, Collins LC: Comparison of molecular phenotypes of ductal carcinoma in situ and invasive breast cancer. Breast Cancer Res 2008, 10:R67.

27. Van de Vijver MJ: Biological variables and prognosis of DCIS. Breast 2005, 14:509-519.

28. Wang S-Y, Shamliyan T, Virnig BA, Kane R: Tumor characteristics as predictors of local recurrence after treatment of ductal carcinoma in situ: a meta-analysis. Breast Cancer Res Treat 2011, 127:1-14.

29. Wapnir IL, Dignam JJ, Fisher B, Mamounas EP, Anderson SJ, Julian TB, Land SR, Margolese RG, Swain SM, Constantino JP, Wolmark N: Long-term outcomes of invasive ipsilateral breast tumor recurrences after lumpectomy in NSABP B-17 and B-24 randomized clinical trials for DCIS. J Natl Cancer Inst 2011, 103:478-488.

30. Benson JR, Wishart GC: Predictors of recurrence for ductal carcinoma in situ after breast-conserving surgery. Lancet Oncol 2013, 14:348-357.

31. Gomes DS, Porto SS, Balabram D, Gobbi H: Inter-observer variability between general pathologists and a specialist in breast pathology in the diagnosis of lobular neoplasia, columnar cell lesions, atypical ductal hyperplasia and ductal carcinoma in situ of the breast. Diagn Pathol 2014, 9:121.

32. Salles M, de A, Sanches FSF, Perez AA, Gobbi H: Importance of a second opinion in breast surgical pathology and therapeutic implications. Rev Bras Ginecol Obstet 2008, 30:602-608.

33. Perez AA, Balabram D, Salles M, de A, Gobbi H: Consultation in breast surgical pathology: interobserver diagnostic variability of atypical intraductal proliferative lesions. Rev Bras Ginecol Obstet 2013, 35:164-170.

34. Silverstein MJ: The University of Southern California/Van Nuys prognostic index for ductal carcinoma in situ of the breast. Am J Surg 2003, 186:337-343.

35. Goldstein NS, Kestin L, Vicini F: Intraductal carcinoma of the breast: pathologic features associated with local recurrence in patients treated with breast-conserving therapy. Am J Surg Pathol 2000, 24:1058-1067.

doi:10.1186/s13000-014-0227-3

Cite this article as: Perez et al:: Ductal carcinoma in situ of the breast: correlation between histopathological features and age of patients. Diagnostic Pathology 2014 9:227.

\section{Submit your next manuscript to BioMed Central and take full advantage of:}

- Convenient online submission

- Thorough peer review

- No space constraints or color figure charges

- Immediate publication on acceptance

- Inclusion in PubMed, CAS, Scopus and Google Scholar

- Research which is freely available for redistribution 